\title{
A GAME WITH NO SOLUTION ${ }^{1}$
}

\author{
W. F. LUCAS
}

Communicated by R. Creighton Buck, November 1, 1967

1. Introduction. In 1944 von Neumann and Morgenstern [2] introduced a theory of solutions for $n$-person games in characteristic function form. The main mathematical question concerning their model is whether every game has at least one solution. This announcement describes a ten-person game which has no solution. The essential definitions for an $n$-person game will be reviewed briefly before the particular example is given. The proof that the game has no solution will then be sketched; a detailed proof will be published elsewhere.

2. Definitions. An $n$-person game is a pair $(N, v)$ where $N$ $=\{1,2, \cdots, n\}$ is the set of players and $v$ is a characteristic function on $2^{N}$, i.e., $v$ assigns the real number $v(S)$ to each subset $S$ of $N$ and $v(\phi)=0$. The set of imputations is

$$
A=\left\{x: \sum_{i \in N} x_{i}=v(N) \text { and } x_{i} \geqq v(\{i\}) \text { for all } i \in N\right\}
$$

where $x=\left(x_{1}, x_{2}, \cdots, x_{n}\right)$ is a vector with real components. For any $X \subset A$ and nonempty $S \subset N$, define $\operatorname{Dom}_{S} X$ to be the set of all $x \in A$ such that there exists a $y \in X$ with $y_{i}>x_{i}$ for all $i \in S$ and with $\sum_{i \in S} y_{i} \leqq v(S)$. Let Dom $X=\cup_{S \subset N} \operatorname{Dom}_{S} X$. Also let $\operatorname{Dom}^{-1} X$ be the set of all $y \in A$ such that there exists $x \in X$ with $x \in \operatorname{Dom}\{y\}$. A subset $K$ of $A$ is a solution if $K \cap \operatorname{Dom} K=\phi$ and $K \cup \operatorname{Dom} K=A$. If $X \subset A$ and $K^{\prime} \subset X$, then $K^{\prime}$ is a solution for $X$ if $K^{\prime} \cap D o m K^{\prime}=\phi$ and $K^{\prime} \cup$ Dom $K^{\prime} \supset X$. The core of a game is

$$
C=\left\{x \in A: \sum_{i \in S} x_{i} \geqq v(S) \text { for all } S \subset N\right\} .
$$

For any solution $K, C \subset K$ and $K \cap \operatorname{Dom} C=\phi$.

A characteristic function $v$ is superadditive if $v\left(S_{1} \cup S_{2}\right) \geqq v\left(S_{1}\right)$ $+v\left(S_{2}\right)$ whenever $S_{1} \cap S_{2}=\phi$. The game listed below does not have a superadditive $v$ as assumed in the classical theory. However, it is equivalent solutionwise to a game with a superadditive $v$. (See Gillies $[1$, p. 68].)

1 This research is sponsored by the United States Air Force under Project RAND. 
3. Example. Consider the game $(N, v)$ where $N=\{1,2,3,4,5,6$, $7,8,9,10\}$ and $v$ is given by:

$$
\begin{aligned}
& v(N)=5, v(\{1,3,5,7,9\})=4, \\
& v(\{1,2\})=v(\{3,4\})=v(\{5,6\})=v(\{7,8\})=v(\{9,10\})=1, \\
& v(\{3,5,7,9\})=v(\{1,5,7,9\})=v(\{1,3,7,9\})=3, \\
& v(\{3,5,7\})=v(\{1,5,7\})=v(\{1,3,7\})=2, \\
& v(\{3,5,9\})=v(\{1,5,9\})=v(\{1,3,9\})=2, \\
& v(\{1,4,7,9\})=v(\{3,6,7,9\})=v(\{5,2,7,9\})=2, \\
& v(S)=0 \text { for all other } S \subset N .
\end{aligned}
$$

For this game

$$
A=\left\{x: \sum_{i \in N} x_{i}=5 \text { and } x_{i} \geqq 0 \text { for all } i \in N\right\} .
$$

One can also show that $C$ is the convex hull of the six imputations:

$(1,0,1,0,1,0,1,0,1,0),(0,1,1,0,1,0,1,0,1,0),(1,0,0,1,1,0,1,0,1,0)$,

$(1,0,1,0,0,1,1,0,1,0),(1,0,1,0,1,0,0,1,1,0)$, and

$(1,0,1,0,1,0,1,0,0,1)$.

4. Outline of proof. Consider the following subsets of $A$ :

$$
\begin{aligned}
B= & \left\{x \in A: x_{1}+x_{2}=x_{8}+x_{4}=x_{5}+x_{6}=x_{7}+x_{8}=x_{9}+x_{10}=1\right\}, \\
E_{i}= & \left\{x \in B: x_{j}=x_{k}=1, x_{i}<1, x_{7}+x_{9}<1\right\}, \\
E= & U_{i} E_{6}, \quad i=1,3,5, \\
F= & {\left[\bigcup_{(j, k)}\left\{x \in B: x_{j}=x_{k}=1, x_{7}+x_{9} \geqq 1\right\}\right.} \\
& \cup U_{(p, q)}\left\{x \in B: x_{p}=1, x_{q}<1, x_{8}+x_{5}+x_{q} \geqq 2,\right. \\
& \left.x_{1}+x_{5}+x_{q} \geqq 2, x_{1}+x_{8}+x_{q} \geqq 2\right\} \\
& \left.\cup\left\{x \in B: x_{7}=x_{9}=1\right\} \cup\left\{x \in B: x_{1}=x_{8}=x_{5}=1\right\}\right]-C,
\end{aligned}
$$

where $(i, j, k)=(1,3,5),(3,5,1)$, and $(5,1,3) ;$ and $(p, q)=(7,9)$ and $(9,7)$. One can verify that the subsets $A-B, B-(C \cup E \cup F), C, E$, and $F$ form a partition of $A$.

To prove that this game has no solution it is sufficient to prove that

(1) Dom $C \supset[A-B] \cup[B-(C \cup E \cup F)]$,

(2) $E \cap \operatorname{Dom}(C \cup F)=\phi$, and

(3) there is no solution for $E$.

One can prove (1) and (2) by checking various subsets $S$ of $N$. In fact, one can prove in addition that Dom $C=A-(C \cup E \cup F)$, and 
$F \cap \operatorname{Dom}(C \cup E \cup F)=\phi$; and thus $C \cup F$ is contained in every solution.

Now consider the region $E$. One can check that $E_{i} \cap \operatorname{Dom}_{S} E=\phi$ for all $S$ except $\{i, r, 7,9\}$, and

$$
E_{i} \cap \operatorname{Dom}_{\{i, r, 7,9\}}\left(E_{i} \cup E_{k}\right)=\phi
$$

where $(i, r, k)=(1,4,5),(3,6,1)$, and $(5,2,3)$. Thus the "Dom" pattern in $E$ is cyclic as illustrated by the diagram:

$$
E_{5} \underset{\{3,6,7,9\}}{ } E_{3} \underset{\{1,4,7,9\}}{\rightarrow} E_{1} \underset{\{5,2,7,9\}}{ } E_{6} \text {. }
$$

To prove (3), assume that $K^{\prime}(\neq \phi)$ is a solution for $E$ and pick any $y \in K^{\prime}$. Using the symmetry in $E$, one can assume $y \in E_{3}$. Define

$$
G_{i}(y)=\left\{x \in E_{i}: x_{7}>y_{7}, x_{9}>y_{9}, x_{k}+x_{r}+x_{7}+x_{9} \leqq 2\right\}
$$

where $(i, k, r)=(1,5,2),(3,1,4)$, and $(5,3,6)$. Then one can verify that $E \cap \operatorname{Dom}^{-1}\{y\}=G_{5}(y)$, and so $K^{\prime} \cap G_{5}(y)=\phi$. However, $E \cap \operatorname{Dom}^{-1} G_{5}(y)=G_{1}(y)$, and so

$$
K^{\prime} \cap G_{1}(y) \neq \phi .
$$

On the other hand, $G_{3}(y) \cap \operatorname{Dom}\left(E_{5}-G_{5}(y)\right)=\phi$, and so $G_{3}(y) \subset K^{\prime}$. However, $G_{1}(y) \subset \operatorname{Dom} G_{3}(y)$, and so

$$
K^{\prime} \cap G_{1}(y)=\phi
$$

which gives a contradiction. Therefore, there is no solution $K^{\prime}$ for $E$.

5. Acknowledgment. The author is grateful to L. S. Shapley for many helpful discussions.

\section{REFERENCES}

1. D. B. Gillies, "Solutions to general non-zero-sum games," Annals of Mathematics Studies, No. 40, A. W. Tucker and R. D. Luce (eds.), Princeton Univ. Press, Princeton, N. J., 1959, pp. 47-85.

2. J. von Neumann and $\mathrm{O}$. Morgenstern, Theory of games and economic behavior. Princeton Univ. Press, Princeton, N. J., 1944.

The Rand Corporation, Santa Monica, California 